\title{
Economic evaluation of voriconazole for the treatment of candidemia in Canadian adults
}

\author{
Coleman Rotstein MD FRCPC FACP ${ }^{1}$, Lael Cragin $\mathrm{MPH}^{2}$, Michel Laverdière $\mathrm{MD}^{3}$, Gary Garber MD FRCPC FACP , \\ Eric J Bow MD FRCPC ${ }^{5,6}$, Alissa Scalera $\mathrm{PhD}^{7}$, Craig Roberts PharmD MPA ${ }^{8}$, Sonja V Sorenson $\mathrm{MPH}^{9}$; \\ the Canadian Expert Panel
}

\begin{abstract}
C Rotstein, L Cragin, M Laverdière, et al; the Canadian Expert Panel. Economic evaluation of voriconazole for the treatment of candidemia in Canadian adults. Can J Infect Dis Med Microbiol $2008 ; 19(3): 219-226$.
\end{abstract}

BACKGROUND: Candidemia is a common cause of nosocomial bloodstream infection. When selecting therapeutic treatments for candidemia, cost-effectiveness is an important consideration. The present study assessed the cost-effectiveness of voriconazole for the treatment of candidemia.

METHODS: A decision-analytical model was used for evaluating the cost-effectiveness of voriconazole compared with a regimen of conventional amphotericin B (CAB) followed by fluconazole (FLU) in the treatment of non-neutropenic patients diagnosed with candidemia in the Canadian setting, based on the Global Candidemia Study. The time frame of the model was 98 days ( 14 weeks). Model parameters were based primarily on clinical outcome, and resource use data collected from the clinical trial were used. Supplemental data were obtained from an independent panel of 12 Canadian experts for parameters not available from the clinical trial. Unit costs were collected from Canadian sources. The outcome variables selected in the study were the number of patients cured within 98 days, the number of patients surviving at 98 days and the number of patients avoiding toxicity. Incremental costs per outcome were calculated to compare the cost-effectiveness analyses (both probabilistic and one-way sensitivity analyses were performed).

RESULTS: The cost-effectiveness analysis demonstrated a difference of $\$ 1,121$ in the total average cost of treatment with voriconazole $(\$ 70,489)$ versus $\mathrm{CAB} / \mathrm{FLU}(\$ 69,368)$. While the costs of voriconazole exceeded the costs of $\mathrm{CAB} / \mathrm{FLU}$, these costs were almost completely offset by lower hospitalization costs. While patients in both treatment arms experienced cure rates of $41 \%$, both the percentage of patients surviving at day 98 $(64.5 \%$ versus $58.2 \%)$ and the percentage of patients avoiding toxicity (64.5\% versus $52.5 \%)$ were higher in the voriconazole arm. Accounting for differences in total costs and clinical outcomes, this analysis estimated an incremental cost per patient surviving at day 98 of $\$ 17,739$, and an incremental cost per patient avoiding toxicity of $\$ 9,298$. In the case of cost per patient cured, voriconazole had a higher cost $(\$ 1,121)$ than $\mathrm{CAB} / \mathrm{FLU}$. The results of the deterministic and probabilistic sensitivity analyses indicated that the model was robust.

CONCLUSIONS: Results of the decision-analytical model provided evidence to support the cost-effectiveness of voriconazole relative to a regimen of $\mathrm{CAB} / \mathrm{FLU}$ in the treatment of non-neutropenic patients diagnosed with candidemia in the Canadian setting.

Key Words: Candidemia; Economic evaluation; Voriconazole

\section{L'évaluation économique du voriconazole dans le traitement de la septicémie à Candida chez les adultes canadiens}

HISTORIQUE : La septicémie à Candida est une cause courante
d'infection sanguine nosocomiale. Le rapport coût-efficacité constitue un
aspect important du choix des traitements thérapeutiques de la septicémie à
Candida. La présente étude vise à évaluer le rapport coût-efficacité du
voriconazole dans le traitement de la septicémie à Candida.
MÉTHODOLOGIE : Les auteurs ont utilisé un modèle de décision
analytique pour évaluer le rapport coût-efficacité du voriconazole par rapport
à une posologie d'amphotéricine B classique (ABC) suivie de fluconazole
(FLU) dans le traitement des patients non neutropéniques atteints d'une
septicémie à Candida dans le contexte canadien, d'après la Global
Candidemia Study. Le calendrier du modèle était de 98 jours (14 semaines).
Les paramètres du modèle se fondaient d'abord sur les issues cliniques, et les
auteurs ont utilisé des données sur l'utilisation des ressources tirées de l'essai
clinique. Ils ont obtenu des données supplémentaires auprès d'un groupe
indépendant de 12 experts canadiens à l'égard des paramètres non
disponibles dans l'essai clinique. Les coûts unitaires provenaient de sources
canadiennes. Les variables d'issues sélectionnées dans l'étude s'établissaient
comme suit : nombre de patients survivants à 98 jours et nombre de patients
évitant la toxicité. Les auteurs ont calculé les coûts incrémentiels par issue
pour comparer les analyses coût-efficacité (à la fois à l'aide d'une analyse
probabiliste et d'une analyse de sensibilité unidirectionnelle). RÉSULTATS : L'analyse coût-efficacité a démontré une différence de 1121 \$ dans le coût total moyen du traitement au voriconazole (70 489 \$) par rapport à l'ABC suivie du FLU (69 368 \$). Même si les coûts du voriconazole étaient supérieurs à ceux de l'ABC suivie du FLU, ils étaient presque totalement compensés par les frais d'hospitalisation moins élevés. Les patients des deux volets du traitement ont obtenu des taux de guérison de $41 \%$, le pourcentage de patients survivants à 98 jours $(64,5 \%$ par rapport à $58,2 \%)$ et le pourcentage de ceux évitant la toxicité (64,5\% par rapport à $52,5 \%$ ) étant plus élevés dans le volet du voriconazole. Pour tenir compte des différences de coûts totaux et d'issues cliniques, cette analyse a estimé un coût incrémentiel de 17739 \$ par patient survivant à 98 jours et un coût incrémentiel de 9298 \$ par patient évitant la toxicité. Pour ce qui est du coût par patient guéri, le voriconazole coûtait plus cher (1 121 \$) que l'ABC-FLU. Les résultats des analyses de sensibilité déterministe et probabiliste confirmaient la solidité du modèle.

CONCLUSIONS : Les résultats du modèle d'analyse décisionnelle ont fourni des données probantes pour appuyer le rapport coût-efficacité du voriconazole par rapport à une posologie d'ABC suivie du FLU dans le traitement des patients non neutropéniques atteints d'une septicémie à Candida diagnostiquée dans le contexte canadien.

${ }^{1}$ Division of Infectious Diseases, Department of Medicine, McMaster University, Hamilton, Ontario; ${ }^{2}$ Center for Health Economics, Epidemiology and Science Policy, United BioSource Corporation, Maryland, USA; ${ }^{3}$ Department of Microbiology-Infectious Diseases, Hospital Maisonneauve-Rosemont, University of Montreal, Montreal, Quebec; ${ }^{4}$ Division of Infectious Diseases, University of Ottawa, Ottawa, Ontario; ${ }^{5}$ Department of Internal Medicine and Medical Microbiology, University of Manitoba; ${ }^{6}$ Department of Medical Oncology and Haematology, CancerCare, Winnipeg, Manitoba; ${ }^{7}$ Pfizer Canada Inc, Montreal, Quebec; ${ }^{8}$ Pfizer Inc; ${ }^{9}$ Center for Health Economics, Epidemiology and Science Policy, United BioSource Corporation, Maryland, USA

Correspondence: Dr Coleman Rotstein, University Health Network, Toronto General Hospital, New Clinical Services Building 11-1212,

200 Elizabeth Street, Toronto, Ontario M5G 2C4. Telephone 416-340-4800 ext 6663, fax 416-340-4242, e-mail Coleman.Rotstein@uhn.on.ca Received and accepted for publication February 27, 2008 
C andida species are among the most common causes of hospital-acquired bloodstream infection. In a large database of nosocomial bloodstream infections in the United States, the Candida species ranked fourth overall among the most common nosocomial bloodstream infections, and one-third among infections in the intensive care unit (ICU), accounting for $10.1 \%$ of all ICU bloodstream infections (1). In a survey of all ICUs in the Calgary Health Region (Calgary, Alberta) between May 2000 and April 2003, Candida species accounted for $9.4 \%$ of isolates obtained from patients with bloodstream infections, and were the fourth most common pathogens (2). These Candida bloodstream infections result in considerable morbidity, mortality and excess hospital expenses due to extended length of stay (3-7).

In the past, the choice of antifungal therapy for candidemia has been predicated on the eradication of the organism, clinical efficacy at the end of active therapy and an enhanced safety profile. The guidelines published in 2004 by the Infectious Diseases Society of America (8) listed conventional amphotericin B (CAB), fluconazole (FLU), combination therapy with FLU plus CAB, caspofungin and lipid formulations of amphotericin B as initial therapeutic options for candidemia and invasive candidiasis, based on organism susceptibility and efficacy in these infections. However, clinicians have overwhelmingly gravitated to the use of FLU due to its superior safety and noninferior efficacy profile compared with CAB (9-12), its availability in both intravenous (IV) and oral formulations, and its significant cost advantage over caspofungin and the lipid formulations of amphotericin B (13-15).

With the increasing incidence of bloodstream infections caused by Candida species with reduced susceptibilities to FLU, such as Candida glabrata and Candida krusei, some doubt has arisen as to the advisability of initial FLU therapy for clinically unstable candidemic patients and those individuals in whom the species of Candida is unknown (16-18). One recent study (18) in Canada, in which susceptibility testing was performed on 184 invasive Candida isolates, found that $30 \%$ of isolates demonstrated reduced susceptibility to FLU. These issues have prompted clinicians to explore alternative antifungal therapies for candidemia $(19,20)$.

An alternative for the treatment of candidemia is another triazole - voriconazole. In a randomized, double-blind, multinational phase III clinical trial (Global Candidemia Study), voriconazole proved to be as effective as a regimen, consisting of a short course of CAB followed by FLU for the treatment of candidemia in non-neutropenic adult patients (20). Voriconazole, although efficacious and characterized by a relatively good safety profile, has the disadvantage of higher drug acquisition costs. Conversely, CAB possesses relatively low drug acquisition costs, but the resource utilization costs generated predominantly by renal toxicities associated with $\mathrm{CAB}$ are quite substantial (5). Based on the aforementioned parameters of efficacy and safety, one may question whether voriconazole is cost-effective compared with less expensive alternatives, such as CAB and FLU. Therefore, the purpose of the present investigation is to assess the cost-effectiveness of voriconazole relative to a $\mathrm{CAB} / \mathrm{FLU}$ regimen using data from the Global Candidemia Study (20).

\section{Model approach}

\section{METHODS}

A decision-analytical model was developed to evaluate the cost-effectiveness of voriconazole compared with a regimen of IV CAB followed by FLU in the treatment of nonneutropenic patients with candidemia. The model takes the perspective of the Canadian provincial Ministries of Health, in which only direct medical costs are included, and follows patients over a 98-day period ( 14 weeks) or until death, if it occurs before day 98 . This time frame captures all relevant outcomes, treatment-related expenditures and consequences, and corresponds to that used in the Global Candidemia Study. Because the time frame for the present study was limited to a 98-day period, neither costs nor outcomes were discounted.

Model pathways, clinical outcomes and resource use parameters were based primarily on data collected from the aforementioned Global Candidemia Study, comparing the safety and efficacy of voriconazole (IV and oral) with a regimen of IV CAB followed by FLU for the treatment of patients (12 years of age or older) with candidemia (20). The characteristics of the patients in the two treatment groups were similar in terms of age, sex, illness severity and medical intervention (20). Data for patients with a blood culture positive for Candida species and who received at least one dose of study drug were included in the posthoc analysis. The values for parameters obtained from the clinical trial and used in the model included cure rates, mortality rates, days of initial IV and oral therapy, hospital length of stay for patients completing initial therapy, time to discontinuation of initial therapy, adverse event rates of initial therapies and the percentage of patients receiving mechanical ventilation at baseline.

An independent Expert Panel was surveyed to review and validate the values for parameters based on the Global Candidemia Study, and to supplement any missing data with reasonable values. Twelve Canadian experts (10 physicians and two hospital pharmacists), with experience in managing invasive fungal infections, were first independently surveyed and then met for a face-to-face meeting to achieve consensus on controversial responses. A majority vote of the Panel was required to reach a consensus and to determine values for any missing data. Values for parameters that could not be obtained from the clinical trial were obtained from the Canadian Expert Panel. The Expert Panel rendered decisions applicable to the Canadian environment that included switch therapy following discontinuation; dose and duration of switch therapy; additional hospital length of stay after patients discontinued initial therapy; duration of mechanical ventilation; and resource use related to diagnostic services, fungal cultures, patient monitoring and concomitant medication.

\section{Model structure}

A diagram of the model structure is provided in Figure 1, and describes the combination of clinical events that can occur when treating candidemia with voriconazole or CAB/FLU, not necessarily the sequence of events. The model pathways consider completion of initial therapy, treatment switching, toxicities (ie, renal, hepatic and other), cure and survival.

Patients who discontinued the initial treatment regimen were assumed to switch to a different antifungal agent, 


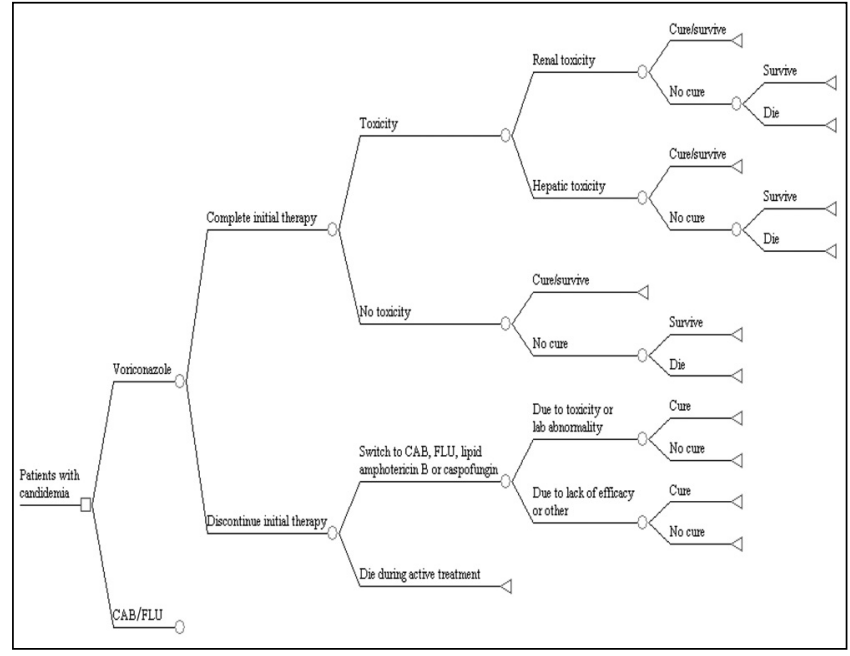

Figure 1) The model structure describes the combination of clinical events that may occur, not necessarily the sequence of the events; all patients who discontinued the initial therapy and switched to another drug survived. The conventional amphotericin B/fluconazole (CAB/FLU) arm is identical to the voriconazole arm, except that patients may only switch to a lipid formulation of amphotericin B or caspofungin. Lab Laboratory

unless death occurred during active initial therapy. Reasons for discontinuation included toxicity (defined as an adverse event or laboratory abnormality), lack of efficacy (defined as an inadequate clinical response, loss to follow-up, protocol violation or withdrawn consent) or other reasons. Renal toxicity was defined as an electrolyte abnormality; hypokalemia; hypomagnesemia; acute kidney failure; kidney function abnormalities; increased urea, oliguria or anuria; or doubling of serum creatinine levels from the baseline level while on treatment. Hepatic toxicity was defined as total bilirubin levels greater than 1.5 times the upper limit of normal (ULN); aspartate aminotransferase (AST), alanine aminotransferase (ALT) or alkaline phosphatase levels greater than three times the ULN; or albumin levels less than 0.8 times the ULN. Other toxicity was defined as any toxicity not meeting the criteria for renal or hepatic toxicity, but which necessitated discontinuation of the initial study drug. Cure was defined as mycological eradication and clinical cure or improvement 12 weeks after completion of therapy, consistent with the primary outcome of the clinical trial.

\section{Model inputs}

Table 1 shows the probability of a patient experiencing each major model pathway, and the associated hospital resource use collected from the Global Candidemia Study and supplemented by the Expert Panel. Patients who discontinued initial treatment and did not die during the 98-day follow-up (Table 2) were assumed to switch to another antifungal agent, and were subsequently cured. According to the Panel, switch therapy was dependent on initial therapy, reason for discontinuation (ie, toxicity, lack of efficacy or other reasons) and type of toxicity (ie, renal, hepatic or other). Table 3 summarizes their recommendations and presents the average daily costs by dose for switch therapies, based on a $67.7 \mathrm{~kg}$ patient, which corresponds to the mean weight of
TABLE 1

Model parameters based on the clinical trial

\begin{tabular}{|c|c|c|c|c|}
\hline & \multicolumn{2}{|c|}{ Voriconazole } & \multicolumn{2}{|c|}{ CAB/FLU } \\
\hline & $\mathbf{n}$ & $\%$ & $\mathbf{n}$ & $\%$ \\
\hline Total & 248 & 100.0 & 122 & 100.0 \\
\hline Completed initial therapy & 145 & 58.5 & 79 & 64.8 \\
\hline Toxicity & 61 & 24.6 & 51 & 41.8 \\
\hline No toxicity & 84 & 33.9 & 28 & 23.0 \\
\hline $\begin{array}{l}\text { Discontinued initial therapy } \\
\text { (switched drug)* }\end{array}$ & 40 & 16.1 & 11 & 9.0 \\
\hline Due to toxicity & 16 & 6.5 & 5 & 4.1 \\
\hline Due to lack of efficacy or othe & r 24 & 9.7 & 6 & 4.9 \\
\hline Died during active treatment & 63 & 25.4 & 32 & 26.2 \\
\hline Mean length of stay & $\begin{array}{l}\text { ICU } \\
\text { days }\end{array}$ & $\begin{array}{c}\text { Total } \\
\text { hospital } \\
\text { days }\end{array}$ & $\begin{array}{l}\text { ICU } \\
\text { days }\end{array}$ & $\begin{array}{c}\text { Total } \\
\text { hospital } \\
\text { days }\end{array}$ \\
\hline \multicolumn{5}{|l|}{ Completed initial therapy } \\
\hline Toxicity & 12.7 & 41.9 & 10.9 & 42.8 \\
\hline No toxicity & 9.3 & 37.6 & 12.5 & 38.5 \\
\hline \multicolumn{5}{|l|}{ Discontinued initial therapy ${ }^{\dagger}$} \\
\hline Due to toxicity & 11.4 & 59.7 & 17.9 & 66.1 \\
\hline Due to lack of efficacy & 13.5 & 58.5 & 28.3 & 67.5 \\
\hline
\end{tabular}

*It was assumed that patients who discontinued initial treatment and did not die during the 98-day follow-up, switched to another therapy (or combination therapy), and were all cured. Includes all patients (completers and discontinuers of initial therapy). ${ }^{t}$ Due to missing follow-up data, the Expert Panel assigned additional length of stay for discontinuers based on trial data for completers. For patients discontinuing initial therapy due to toxicity, additional length of stay was based on data from completers who developed toxicity and survived, whereas length of stay for patients discontinuing due to lack of efficacy was based on data from all completers who survived. CAB/FLU Conventional amphotericin B/fluconazole; ICU Intensive care unit

patients in the Global Candidemia Study. Patients discontinuing voriconazole switched to CAB, FLU, a lipid formulation of amphotericin B (ie, AmBisome [Astellas Pharma, Canada] or ABELCET [Enzon Pharmaceuticals Inc, USA]) or caspofungin. Patients discontinuing the CAB/FLU regimen switched to a lipid formulation of amphotericin B or caspofungin. Based on expert opinion, patients discontinuing initial therapy due to lack of efficacy were assumed to have received no clinical benefit and, thus, were assigned a full course of antifungal switch therapy (14 days). Despite their adverse events, patients discontinuing therapy due to toxicity were assumed to have received some clinical benefit and, based on expert opinion, were assigned only 9.6 days of additional antifungal switch therapy, which ensured that patients completed the equivalent of an average course of therapy. Thus, the assignment of 9.6 days of therapy by the Expert Panel was based on the average duration of therapy minus the average duration of therapy before the initiation of the switch therapy.

Drug costs were collected from publicly available Canadian sources except for the unit cost of voriconazole, which was collected from Pfizer Canada Inc. Table 2 presents the doses and weighted average costs per day, as well as the mean duration of initial drug therapy. The weighted average costs per day of treatment were based on the number of vials (or tablets) required by patients, as determined by the weight distribution of patients in the clinical trial. 
TABLE 2

Weighted average cost per day and mean duration of initial drug therapy

\begin{tabular}{|c|c|c|c|c|c|}
\hline & \multirow{2}{*}{\multicolumn{2}{|c|}{ Voriconazole }} & \multicolumn{3}{|c|}{ CAB/FLU } \\
\hline & & & \multirow{2}{*}{$\begin{array}{c}\text { CAB } \\
\text { IV }\end{array}$} & \multicolumn{2}{|c|}{ FLU } \\
\hline & IV & Oral & & IV & Oral \\
\hline Dose (mg/kg) & $3-6$ & $200-400$ & 1 & 400 & 400 \\
\hline $\begin{array}{l}\text { Weighted average } \\
\text { cost per day }(\$)\end{array}$ & $411-709$ & 93 & 71 & 73 & 22 \\
\hline \multicolumn{6}{|c|}{ Mean duration of initial drug therapy (days) } \\
\hline \multicolumn{6}{|l|}{ Completed initial therapy } \\
\hline Toxicity & 12.8 & 7.3 & 6.5 & 6.8 & 7.3 \\
\hline No toxicity & 11.3 & 8.5 & 5.3 & 7.4 & 6.7 \\
\hline \multicolumn{6}{|l|}{ Discontinued initial therapy } \\
\hline Due to toxicity & 7.4 & 1.2 & 3.2 & 1.4 & 0.8 \\
\hline $\begin{array}{l}\text { Due to lack of } \\
\text { efficacy or other }\end{array}$ & 11.5 & 4.0 & 4.7 & 5.0 & 3.0 \\
\hline Died during active treatment & 8.0 & 0.4 & 4.5 & 4.2 & 0.2 \\
\hline
\end{tabular}

The base-case analysis assumes that any unused drug remaining after administration was discarded. Because conventional amphotericin $B(C A B)$ is indicated for several conditions other than candidemia, a sensitivity analysis was performed, in which any unused $C A B$ remaining after administration was recycled for future use. Because the base-case analysis assumes any unused $C A B$ remaining after administration was discarded, the choice between using a CAB dose of $0.7 \mathrm{mg} / \mathrm{kg}$ or $1.0 \mathrm{mg} / \mathrm{kg}$ is of negligible importance. Under both dosing scenarios, the weighted average number of vials required for each patient is two vials per day. FLU Fluconazole; IV Intravenous

ICU status and duration of hospitalization were collected from the clinical trial for all patients during their participation in the study. For patients who discontinued the study, additional resource use was added based on recommendations of the Expert Panel. Per diem costs were $\$ 2,909$ and $\$ 1,279$ for the ICU and non-ICU services, respectively, based on costs from Sunnybrook Health Sciences Centre (Toronto, Ontario), Vancouver Coastal (Vancouver, British Columbia) and Alberta Health Authority (includes physician consultation costs for critical care [day 2 onwards] from the Ontario Schedule of Benefits for Physician Services, October 1, 2005 [21]).

Other resource use and costs

Other resource use considered in the model included fungal screening, mechanical ventilation, concomitant drugs and monitoring over the 98-day study period. Concomitant drugs (ie, hydrocortisone, acetaminophen, diphenhydramine, meperidine, apo-amiloride $\mathrm{HCl}$ and sodium loading) were assigned to $50 \%$ of patients before administration of $\mathrm{CAB}$, $25 \%$ before administration of Abelcet and 20\% before administration of AmBisome, based on clinical experience input from the Expert Panel. Resource use related to monitoring included complete blood counts (including a specimen handling fee) and liver function tests three times per week. Renal function tests were provided daily for patients in the ICU or for patients with renal toxicity, regardless of ward location. Patients not hospitalized in the ICU were ascribed three renal function tests per week. Table 4 presents unit costs collected from publicly available Canadian provincial sources for diagnostic services, fungal screening and mechanical ventilation. The Expert Panel provided estimates of use for these resources.
TABLE 3

Switch therapy, dose and duration of initial therapy, as well as the reason for discontinuation

\begin{tabular}{|c|c|c|c|c|c|}
\hline & $\begin{array}{l}\text { Voriconazole } \\
\qquad(n=40) \\
(\%)\end{array}$ & $\begin{array}{c}\text { CABI } \\
\text { FLU } \\
(n=11) \\
(\%)\end{array}$ & $\begin{array}{r}\text { A } \\
\text { da }\end{array}$ & $\begin{array}{l}\text { Average } \\
\text { daily cost } \\
\text { (\$) }\end{array}$ & $\begin{array}{c}\text { Duration } \\
\text { (days) }\end{array}$ \\
\hline \multicolumn{6}{|c|}{ Discontinued initial therapy - due to toxicity } \\
\hline $\begin{array}{l}\text { Lipid formulation } \\
\text { of amphotericin } B\end{array}$ & B & - & - & - & - \\
\hline AmBisome & 3 & - & $3 \mathrm{mg} / \mathrm{kg}$ & $1,050^{*}$ & 9.6 \\
\hline Abelcet & 3 & - & $3 \mathrm{mg} / \mathrm{kg}$ & $690^{\dagger}$ & 9.6 \\
\hline \multirow[t]{2}{*}{ Caspofungin IV } & - & - & $70 \mathrm{mg}$ load & $567^{\ddagger}$ & 1.0 \\
\hline & 34 & 100 & $50 \mathrm{mg}$ monitor & r $440^{\ddagger}$ & 8.6 \\
\hline $\mathrm{CAB}$ & 32 & - & $0.7 \mathrm{mg} / \mathrm{kg}$ & $37 \S$ & 9.6 \\
\hline FLU & - & - & 200 mgף & $37^{\star *}-11^{\S}$ & 9.6 \\
\hline IV to oral & 14 & - & $400 \mathrm{mg} \pi$ & $73^{\star *}-22^{\S}$ & 9.6 \\
\hline \multicolumn{6}{|c|}{ Discontinued initial therapy - due to lack of efficacy } \\
\hline $\begin{array}{l}\text { Lipid formulation } \\
\text { of amphotericin B }\end{array}$ & $B^{-}$ & - & - & - & - \\
\hline AmBisome & 15 & 5 & $5 \mathrm{mg} / \mathrm{kg}$ & $1,470^{*}$ & 14.0 \\
\hline Abelcet & 15 & 5 & $5 \mathrm{mg} / \mathrm{kg}$ & $920^{\dagger}$ & 14.0 \\
\hline \multirow[t]{2}{*}{ Caspofungin IV } & - & - & $70 \mathrm{mg}$ load & $567^{\ddagger}$ & 1.0 \\
\hline & 40 & 90 & 50 mg monitor & r $440^{\ddagger}$ & 13.0 \\
\hline CAB & 30 & - & $1.0 \mathrm{mg} / \mathrm{kg}$ & $75 \S$ & 14.0 \\
\hline
\end{tabular}

*PPS Pharma 2002 (26); †'Average cost: Enzon Pharmaceuticals Inc (USA) British Columbia and Ontario formularies, and Vancouver coastal pharmacies, ${ }_{\ddagger}$ Alberta Health and Wellness Drug Benefit List (27), October 1, 2005; \$Ontario Drug Benefit Formulary 2005 (Edition 39) (28); TPatients with renal toxicity receive $200 \mathrm{mg}$ of fluconazole (FLU), whereas patients with hepatic toxicity receive $400 \mathrm{mg}$ of $\mathrm{FLU} ;{ }^{* *} Q u e b e c$ Hospital Association. CAB Conventional amphotericin B; IV Intravenous

\section{Model outcomes}

The model was designed to capture both clinical and economic outcomes. The key cost outcome was the total average cost of care per patient for each treatment arm. The effectiveness outcomes selected in the present study were the number of patients surviving at day 98 , the number of patients cured at day 98 and the number of patients avoiding toxicity. All outcomes variables were collected directly from analyses of the clinical trial data.

\section{Analyses}

Resources were multiplied by unit costs for the treatment pathway and then summed to estimate total cost and outcomes for each treatment arm. The primary cost-effectiveness measure was the incremental cost per patient surviving at 98 days. Secondary cost-effectiveness measures were the incremental cost per patient cured and the incremental cost per toxicity avoided. These incremental cost-effectiveness ratios (ICERs) were calculated as the difference in the total average costs of care associated with each treatment regimen divided by the difference in health benefits associated with each treatment regimen (ie, survival, cure and toxicity avoided). The analyses were conducted in Microsoft Excel (Microsoft Corporation, USA).

One-way sensitivity analyses were conducted to identify the primary sources of sensitivity in the model's estimation of treatment costs and outcome measures associated with voriconazole relative to $\mathrm{CAB} / \mathrm{FLU}$. ICU and non-ICU per 
TABLE 4

Diagnostic services, fungal screening and mechanical ventilation costs

\begin{tabular}{|c|c|}
\hline Resource & Unit cost (\$) \\
\hline \multicolumn{2}{|l|}{ Diagnostic services (per initial seven-day period only) } \\
\hline Chest $x$-ray ${ }^{\star}+$ & 21.05 \\
\hline Computed tomography scan*† & 65.90 \\
\hline Ultrasound (abdominal/pelvis) ${ }^{\star \dagger}$ & 210.60 \\
\hline Nephrology consultation*† & 127.50 \\
\hline Gastroenterology consultation ${ }^{\star} \dagger$ & 127.50 \\
\hline \multicolumn{2}{|l|}{ Fungal screening (per initial seven-day period only) } \\
\hline Fungal culture - nonblood ${ }^{\dagger}$ & 12.93 \\
\hline Fungal culture - blood ${ }^{\dagger}$ & 15.51 \\
\hline Fungal culture, $\mathrm{KOH}$ preparation (for blood culture only) ${ }^{\ddagger}$ & 10.34 \\
\hline \multicolumn{2}{|l|}{ Mechanical ventilation } \\
\hline Cost per day of ventilation§ & 89.70 \\
\hline \multicolumn{2}{|c|}{$\begin{array}{l}\text { *Ontario Schedule of Benefits for Physician Services October 1, } 2005 \text { (21); } \\
\text { †To account for fungal screening during the first seven days following diagno- } \\
\text { sis. The analysis includes the cost of one chest x-ray, seven blood cultures, } \\
1.5 \text { nonblood cultures and } 0.5 \text { computed tomography scans. In addition, } 30 \% \\
\text { of patients with renal toxicity require one ultrasound and nephrology consulta- } \\
\text { tion, while } 30 \% \text { of patients with hepatic toxicity require one ultrasound and gas- } \\
\text { troenterology consultation; } ¥ \text { Ontario Schedule of Laboratory Fees: April } 1 \text {, } \\
1999 \text {; §Average Quebec, Ontario and British Columbia Schedule of Benefits. } \\
\text { The duration of mechanical ventilation is assumed to be equivalent to the } \\
\text { number of intensive care unit days minus } 1.5 \text { days. KOH Potassium hydroxide }\end{array}$} \\
\hline
\end{tabular}

diem costs were varied by $\pm 50 \%$, mean weight was varied by $\pm 10 \%$, monitoring costs were varied by $\pm 20 \%$ and the number of voriconazole vials required per day were varied from four to six for loading and from two to three for maintenance. The model was also run assuming $100 \%$ of patients discontinuing initial therapy switched to caspofungin, assuming $100 \%$ switched to a lipid formulation of amphotericin B, assuming any remaining $\mathrm{CAB}$ was not wasted, assuming additional length of hospital stay for discontinuers was decreased by $50 \%$ and assuming a model time frame of 42 days (six weeks) rather than 98 days (14 weeks).

A probabilistic sensitivity analysis using a Monte Carlo simulation was performed to evaluate the impact of simultaneous variation in clinical outcome and resource utilization parameters on the model conclusions. Probabilistic sensitivity analysis involved specifying distributions for model parameters to account for uncertainty in their estimation, and using Monte Carlo simulation to randomly sample each of the parameter distributions and calculate the expected costs and clinical outcomes for that combination of parameter values. This allowed for a range of possible outcomes to be estimated, reflecting uncertainty in the cost-effectiveness ratio. Parameter distributions were defined based on the means and standard deviations from the Global Candidemia Study and other source materials. Cost-effectiveness scatter plots were generated to illustrate the impact of overall uncertainty in the model parameters on the model conclusions. In addition, a net-benefit acceptability curve was used to summarize the results of the cost-effectiveness analysis. Conditional on knowing the maximum amount a payer is willing to pay (threshold value) for a one unit gain in health outcome (for example, a patient surviving until day 98), there is only one treatment of choice from the two strategies under evaluation (ie, the treatment with the greatest
TABLE 5

Total and incremental costs (\$) by drug regimen

\begin{tabular}{lrrr}
\hline & Voriconazole & CAB/FLU & Incremental \\
\hline Total average cost & 70,489 & 69,368 & 1,121 \\
Drug costs for initial therapy & 5,147 & 928 & 4,218 \\
Drug costs for switch therapy & 776 & 540 & 237 \\
Monitoring & 954 & 1,057 & -103 \\
Screening & 287 & 302 & -14 \\
Hospitalization & 62,993 & 66,108 & $-3,115$ \\
Mechanical ventilation & 323 & 370 & -47 \\
Concomitant medications & 9 & 64 & -54 \\
\hline
\end{tabular}

CAB/FLU Conventional amphotericin B/fluconazole

average incremental net-benefit). The number of times that voriconazole is the treatment of choice from the 1000 replications of the model provides an estimate of the strength of evidence in favour of that treatment (22). Although the maximum amount a payer is willing to pay for an additional unit of health benefit was not known, the number of times voriconazole had the greatest net-benefit for all possible threshold values was plotted. Thereafter, a net-benefit acceptability curve was used to understand the probability of voriconazole being the most cost-effective option across a range of a payer's possible willingness to pay for that outcome.

\section{RESULTS}

The results of the analysis indicate a difference of $\$ 1,121$ in the total average cost of treatment with voriconazole $(\$ 70,489)$ versus CAB followed by FLU $(\$ 69,368)$ (Table 5). While patients in the Global Candidemia Study experienced essentially equivalent cure rates at the primary end point (41\%; 101 of 248 versus 50 of 122$)$, an additional $6.3 \%$ (160 of 248 [64.52\%] versus 71 of 122 [58.20\%]) of voriconazole patients survived at day 98 and an additional 12.1\% (160 of 248 [64.52\%] versus 64 of 122 [52.46\%]) avoided toxicity (Figure 2). Accounting for both differences in total costs and clinical outcomes, the analysis estimated an incremental cost per (additional) patient surviving at day 98 of $\$ 17,739$ ([\$70,489-\$69,368]/[0.64516129-0.582196721]), and an incremental cost per (additional) patient avoiding toxicity of \$9,298 ([\$70,489-\$69,368]/[0.64516129-0.52459016]). Considering cost per patient cured, the incremental cost per patient cured was not calculated because voriconazole had a slightly higher cost than $\mathrm{CAB} / \mathrm{FLU}$ and cure rates were equivalent in both arms.

\section{One-way sensitivity analyses}

Figure 3 presents, in decreasing order, the parameters generating the most variation in the model with respect to the incremental cost per additional patient surviving 98 days. The model is most sensitive to the model time frame, ICU per diem costs and the estimated additional length of hospital stay for discontinuers. The 42-day time frame analysis results in the largest ICER due, in part, to the fact that the incremental difference in the percentage of patients surviving at day 42 (which was greater with voriconazole therapy) was approximately one-half the incremental difference in the percentage of patients surviving at day 98. Similar results (not presented) were seen when the impact of the model 


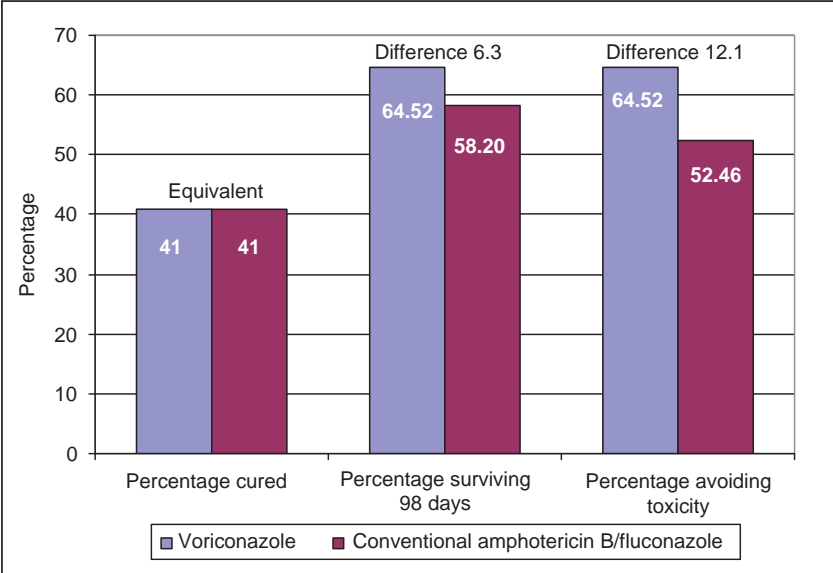

Figure 2) Total and incremental effectiveness of treatment using voriconazole versus conventional amphotericin B/fluconazole. Probabilities do not add to $100 \%$ because a patient could be represented in more than one outcome (eg, patient cured and avoided toxicity)

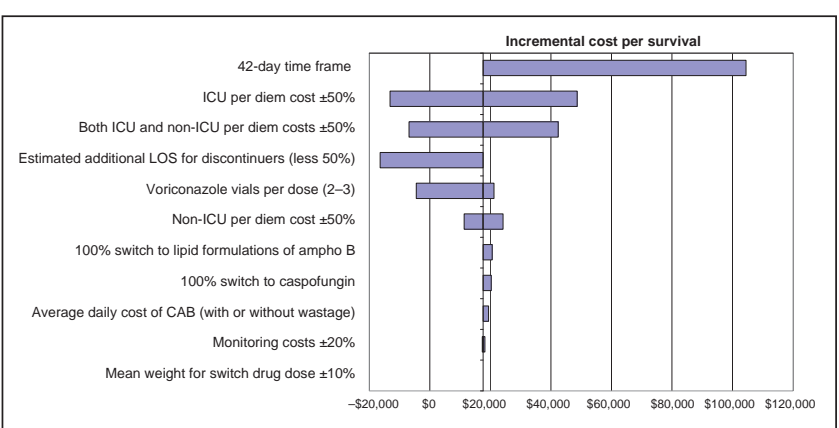

Figure 3) Sensitivity to variation in key model parameters: Incremental cost per survival. $\mathrm{CAB}$ Conventional amphotericin $B$ (ampho B); ICU Intensive care unit; LOS Length of stay

parameters on the incremental cost per patient avoiding toxicity and on the incremental cost per patient cured were assessed.

\section{Probabilistic sensitivity analyses}

Results of the probabilistic sensitivity analysis for patient survival at day 98 (Figure 4) indicate that voriconazole was associated with increased survival at day 98 in $88 \%$ of the simulations, and was less costly in $49 \%$ of the simulations relative to $\mathrm{CAB} / \mathrm{FLU}$; only in a small number of simulations did voriconazole result in both decreased survival at day 98 and increased costs relative to $\mathrm{CAB} / \mathrm{FLU}$. The results of the net-benefit acceptability curve indicate that as willingnessto-pay threshold values for the cost per additional (statistical) life saved by the payer increases, there is progressively greater certainty that voriconazole is cost-effective relative to $\mathrm{CAB} / \mathrm{FLU}$ (Figure 5). Only at maximum willingness-to-pay threshold values less than $\$ 20,000$ per additional life saved does the strength of evidence indicate voriconazole is costeffective less than $50 \%$ of the time.

\section{DISCUSSION}

The present study evaluated the cost-effectiveness of voriconazole relative to $\mathrm{CAB} / \mathrm{FLU}$ in the treatment of

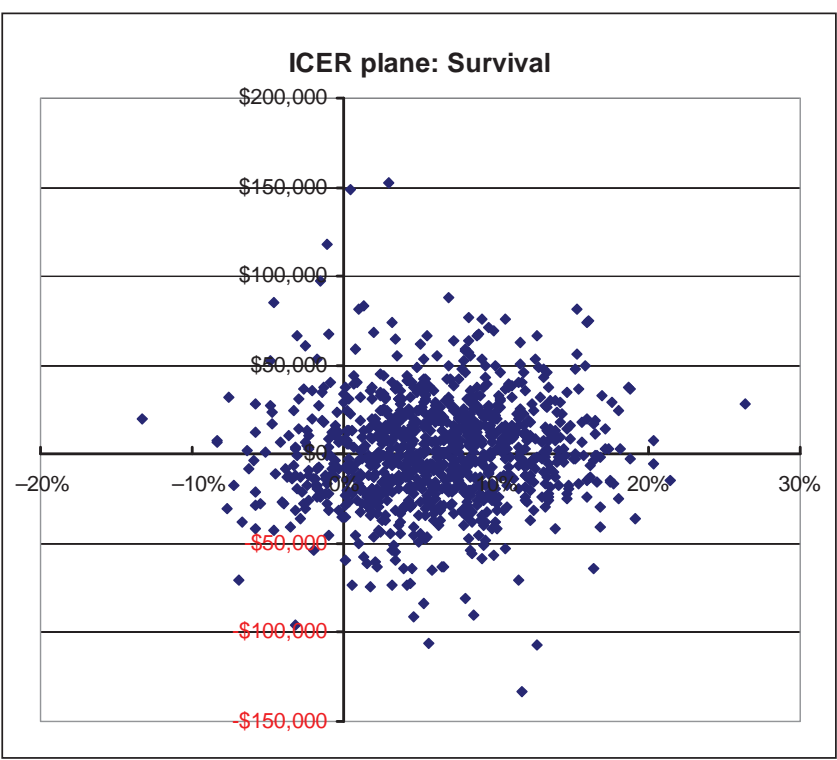

Figure 4) Incremental costs and per cent of patients surviving at 98 days for voriconazole versus conventional amphotericin $B(C A B) /$ fluconazole (FLU). Points to the right of the $y$-axis indicate voriconazole is more effective than $\mathrm{CAB} / \mathrm{FLU}$. Points below the $x$-axis indicate voriconazole is less costly than CAB/FLU. ICER Incremental cost-effectiveness ratios

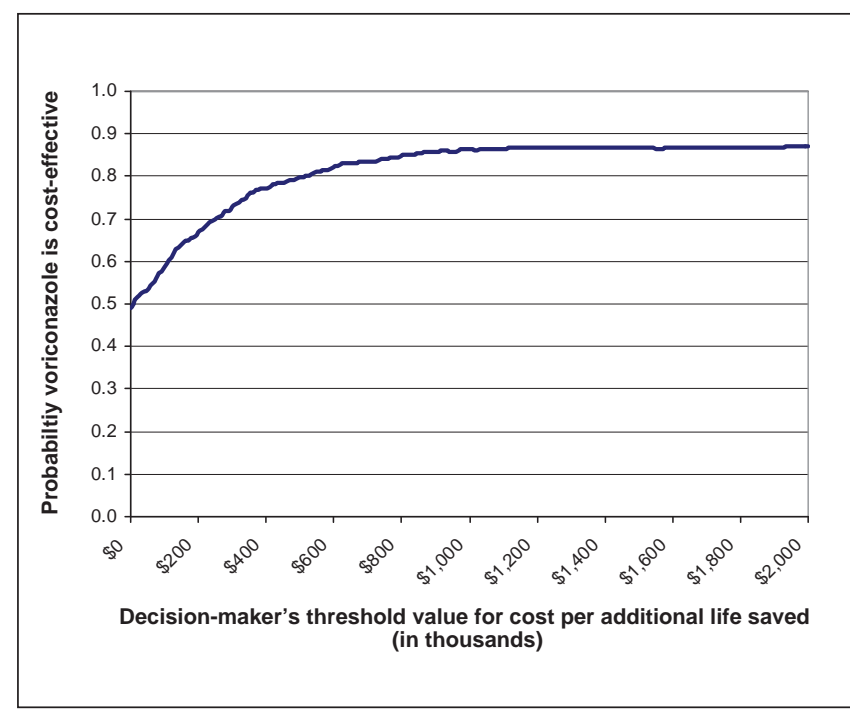

Figure 5) Net-benefit acceptability curve

non-neutropenic patients with candidemia, in the context of limited exposure to $\mathrm{CAB}$ toxicities and the use of oral step-down agents; it was primarily based on data from the Global Candidemia Study. Economic analyses based on clinical trial data are useful in providing decision-makers with information on the expected costs associated with a new therapy at times when 'real world' data may not be available. Supplemental data for parameters that could not be obtained from the trial were collected from an independent Expert Panel of 12 Canadian physicians and hospital pharmacists, who had experience in managing invasive fungal infections. 
This economic evaluation demonstrated that although voriconazole drug acquisition costs exceed both IV CAB and sequential IV or oral FLU, the difference in the total cost of treatment for the Canadian setting over a 98-day time horizon was only $\$ 1,121$ (voriconazole $\$ 70,489$ versus CAB/FLU $\$ 69,368)$, largely due to cost offsets associated with lower hospitalization costs. In addition, when factoring in the clinical outcomes of mortality and toxicity, the additional cost is outweighed by a survival difference of $6.3 \%$ in favour of voriconazole, and a diminished toxicity profile difference of $12.1 \%$ in favour of voriconazole. This is reflected in the modest incremental cost per additional patient surviving at day $98(\$ 17,739)$ and the incremental cost per additional patient avoiding toxicity $(\$ 9,298)$.

As is evident in the results of the present analysis (Table 5), hospitalization costs, not drug costs, account for the majority of direct medical costs associated with treating patients with candidemia. For patients in the CAB/FLU treatment arm, hospitalization costs represented 95\% $(\$ 66,108 / \$ 69,368)$ of total treatment costs, whereas they represented $89 \%(\$ 62,993 / \$ 70,489)$ of total treatment costs for patients in the voriconazole treatment arm $\left(\chi^{2}=1734.5\right.$; $\mathrm{P}<0.001)$. These findings are consistent with the conclusions of other studies $(4,6,7)$, which suggest that the major cost associated with candidemia is that of significantly increased hospital stay. An American study by Rentz et al (6) concluded that patients with candidemia had an average of 34 additional days of hospitalization compared with control patients. Morgan et al (4) reported that patients who received adequate treatment for candidemia were found to have approximately three to 13 more hospitalization days and US $\$ 3,337$ to US $\$ 22,228$ more in hospital costs than matchcontrols. Zaoutis et al (7) estimated that candidemia was associated with a mean 10.1-day increase in length of stay in adult patients, and a mean increase in hospital charges of US $\$ 39,331$.

The results of the deterministic and probabilistic sensitivity analyses indicate that the model, overall, is robust. Moreover, the results underscore the influence of hospitalization costs. According to the deterministic sensitivity analysis, the parameters (or scenarios) to which the model is most sensitive include the model time frame, ICU per diem costs and the estimated additional length of hospital stay for discontinuers. The 42-day time frame analysis results in the largest ICER due, in part, to the fact that the incremental difference in the percentage of patients surviving at day 42 (which was greater with voriconazole therapy) was approximately one-half the incremental difference in the percentage of patients surviving at day 98 . This suggests that perhaps due to the severity of underlying illnesses and improvements in their treatment, as well as improvements in the diagnosis and management of complications related to candidemia, a longer time frame is necessary to adequately capture mortality.

The net-benefit acceptability curve used to determine the probability of voriconazole's cost-effectiveness for hypothetical maximum willingness-to-pay threshold values per additional life saved (Figure 5) indicates that even at low willingness-to-pay threshold values for the cost per (statistical) life saved, there is still a high level of certainty that voriconazole is cost-effective relative to $\mathrm{CAB} / \mathrm{FLU}$ (Figure 5). The cost-effectiveness of voriconazole does not indicate cost-saving, but implies good value for money because it enhances quality and duration of life. Only at maximum willingness-to-pay threshold values less than $\$ 20,000$ per additional life saved, the strength of evidence indicates voriconazole is cost-effective less than $50 \%$ of the time. While health economists are accustomed to using a standard threshold value ranging from US $\$ 50,000$ to US $\$ 100,000$ per additional quality-adjusted life year gained $(14,23,24)$, there is no widely accepted standard threshold value for the cost per additional (statistical) life saved, largely due to reluctance in attaching an explicit monetary value to human life. However, an assessment of the economic literature indicates that most Canadian estimates of the value of a statistical life range from US $\$ 3$ million to US $\$ 6$ million (reported in 2000 US\$) (24). With this in mind, at a conservative willingness-to-pay threshold value of approximately $\$ 1$ million per additional life saved, the analysis in Figure 5 suggests that there is an $87 \%$ probability that voriconazole is cost-effective relative to $\mathrm{CAB} / \mathrm{FLU}$.

Certain key limitations of the present analysis deserve mention. First, the observed mortality differences in the Global Candidemia Study were not statistically significant. However, the trial was not designed or powered to detect statistically significant differences in mortality. Second, as in any economic analysis based on trial data, resource utilization may have been somewhat protocol-driven. Third, supplemental resource use estimates based on cost utilization assumptions were provided by the Expert Panel. Although this is an acceptable method, the inputs may be subject to bias. Finally, although our sensitivity analyses were robust, other scenarios such as shifting a portion of inpatient care to the outpatient setting could have been explored. This is particularly pertinent to $\mathrm{CAB} / \mathrm{FLU}$, in which toxicity and lack of efficacy complications led to prolonged hospitalization and higher costs. Due to data limitations, the model was not able to evaluate the cost-effectiveness of voriconazole compared with FLU alone. Such a comparison would be of particular interest because a recent study (14) concluded that FLU was cost-effective relative to $C A B$, lipid formulations of amphotericin $B$ and caspofungin. Nevertheless, although the results of individual randomized clinical trials (10-12) and one meta-analysis (9) have suggested that FLU efficacy is noninferior to $C A B$ in non-neutropenic patients, a recent pooled analysis (25) suggests the possible superiority of $\mathrm{CAB}$ ( $62 \%$ of 345 patients versus $70 \%$ of 343 patients; $\chi^{2}=5.206$; $\mathrm{P}=0.023$; OR 1.47 ; $95 \%$ CI 1.07 to 2.03 ).

\section{CONCLUSION}

Candidemia is associated with increased total hospital costs and length of stay. The present study has demonstrated that although voriconazole treatment costs were slightly more expensive than $\mathrm{CAB} / \mathrm{FLU}$, voriconazole was cost-effective over a 98-day time frame because it conferred a survival advantage and an avoidance of toxicity in the treatment of candidemia. Thus, despite higher acquisition costs, voriconazole may prove to be cost-effective in the treatment of candidemia.

ACKNOWLEDGEMENTS: The authors thank Vincent Raymond for his advice and assistance in completing this manuscript, and Ms Kathleen Dean for her technical support. 


\section{Appendix (Canadian Expert Panel)}

The Canadian Expert Panel convened on December 3, 2005, to review the pharmacoeconomic analysis. The members of the Canadian Expert Panel who were present to review the models and inputs are listed below.

\begin{tabular}{|lll|}
\hline Dr Eric J Bow & Dr Rene Pelletier & Dr Stephen Lapinsky \\
Health Sciences Centre & Hotel Dieu de Quebec du CHUQ & Mount Sinai Hospital \\
820 Sherbrooke Street, Room GD 600 & 11, cote du Palais & 18-214-600 University Avenue \\
Winnipeg, Manitoba R3A 1R0 & Quebec, Quebec G1R 2J6 & Toronto, Ontario M5G 1X5 \\
Dr Ronald Field & Dr Coleman Rotstein & Dr Gary Garber \\
Princess Margaret Hospital & Hamilton Health Sciences & Ottawa Hospital General Campus \\
610 University Avenue & Henderson Site & 501 Smyth Road \\
Toronto, Ontario M5G 2M9 & 711 Concession Street & Ottawa, Ontario K1H 8L6 \\
Mr Alfred Gin & Hamilton, Ontario L8V 1C3 & Dr Stephen Shafran \\
Health Sciences Centre & Dr Stephen Sanche & University of Alberta \\
Department of Pharmaceutical Services & Royal University Hospital & 2E4.16 Walter MacKenzie HSC \\
820 Sherbrooke Street, Room MS 189 & 103 Hospital Street & 8440-112 Street \\
Winnipeg, Manitoba R3A 1R9 & Saskatoon, Saskatchewan S7N 0W8 & Edmonton, Alberta T6G 2B7 \\
Dr Michel Laverdière & Mr Gary Wong & Dr Atul Humar \\
Hopital Maisonneuve-Rosemont & University Health Network & University of Alberta \\
Department Infectious Diseases & Pharmacy Department & Room 4106 RTF \\
5415 boul. De L'Assomption & 200 Elizabeth Street & 8308-114 Street \\
Montreal, Quebec H1T 2M4 & Toronto, Ontario M5G 2C4 & Edmonton, Alberta T6G 2E1 \\
\hline
\end{tabular}

\section{REFERENCES}

1. Wisplinghoff H, Bischoff T, Tallen SM, Seifert H, Wenzel RP, Edmond MB. Nosocomial bloodstream infections in US hospitals: Analysis of 24,179 cases from a prospective nationwide surveillance study. Clin Infect Dis 2004;39:309-17. (Erratums in 2004;39:1093, 2005;40:1077).

2. Laupland KB, Kirkpatrick AW, Church DL, Ross T, Gregson DB. Intensive-care-unit-acquired bloodstream infections in a regional critically ill population. J Hosp Infect 2004;58:137-45.

3. Wey SB, Mori M, Pfaller MA, Woolson RF, Wenzel RP. Hospital-acquired candidemia. The attributable mortality and excess length of stay. Arch Intern Med 1988;148:2642-5.

4. Morgan J, Meltzer MI, Plikaytis BD, et al. Excess mortality, hospital stay, and cost due to candidemia: A case-control study using data from population-based candidemia surveillance. Infect Control Hosp Epidemiol 2005;26:540-7. (Erratum in 2005;26:540-7).

5. Bates DW, Su L, Yu DT, et al. Mortality and costs of acute renal failure associated with amphotericin B therapy. Clin Infect Dis 2001;32:686-93.

6. Rentz AM, Halpern MT, Bowden R. The impact of candidemia on length of hospital stay, outcome, and overall cost of illness. Clin Infect Dis 1998;27:781-8.

7. Zaoutis TE, Argon J, Chu J, Berlin JA, Walsh TJ, Feudtner C. The epidemiology and attributable outcomes of candidemia in adults and children hospitalized in the United States: A propensity analysis. Clin Infect Dis 2005;41:1232-9.

8. Pappas PG, Rex JH, Sobel JD, et al; Infectious Diseases Society of America. Guidelines for the treatment of candidiasis. Clin Infect Dis 2004;38:161-89.

9. Kontoyiannis DP, Bodey GP, Mantzoros CS. Fluconazole vs. amphotericin B for the management of candidaemia in adults: A meta-analysis. Mycoses 2001;44:125-35.

10. Rex J, Bennett JE, Sugar AM, et al. A randomized trial of fluconazole versus amphotericin B for treatment of candidemia in patients without neutropenia. Candidemia Study Group and the National Institute. N Engl J Med 1994;331:1325-30.

11. Anaissie EJ, Darouiche RO, Abi-Said D, et al. Management of invasive candidal infections: Results of a prospective, randomized, multicenter study of fluconazole versus amphotericin B and review of the literature. Clin Infect Dis 1996;23:964-72.

12. Phillips P, Shafran S, Garber G, et al. Multicenter randomized trial of fluconazole versus amphotericin B for treatment of candidemia in non-neutropenic patients. Canadian Candidemia Study Group. Eur J Clin Microbiol Infect Dis 1997;16:337-45.

13. Spellberg BJ, Filler SG, Edwards JE Jr. Current treatment strategies for disseminated candidiasis. Clin Infect Dis 2006;42:244-51.
14. Golan Y, Wolf MP, Pauker SG, Wong JB, Hadley S. Empirical anti-Candida therapy among selected patients in the intensive care unit: A cost-effectiveness analysis. Ann Intern Med 2005;143:857-69.

15. Dranitsaris G, Phillips P, Rotstein C, et al. Economic analysis of fluconazole versus amphotericin B for the treatment of candidemia in non-neutropenic patients. Pharmacoeconomics 1998;13:509-18.

16. Pappas PG, Rex JH, Lee J, et al; NIAID Mycoses Study Group. A prospective observational study of candidemia: Epidemiology, therapy, and influences on mortality in hospitalized adult and pediatric patients. Clin Infect Dis 2003;37:634-43.

17. Nedret Koç A, Kocagöz S, Erdem F, Gündüz Z. Outbreak of nosocomial fungemia caused by Candida glabrata. Mycoses 2002;45:470-5.

18. Laupland KB, Gregson DB, Church DL, Ross T, Elsayed S. Invasive Candida species infections: A 5 year population-based assessment. J Antimicrob Chemother 2005;56:532-7.

19. Mora-Duarte J, Betts R, Rotstein C, et al; Caspofungin Invasive Candidiasis Study Group. Comparison of caspofungin and amphotericin B for invasive candidiasis. N Engl J Med 2002;347:2020-9.

20. Kullberg BJ, Sobel JD, Ruhnke M, et al. Voriconazole versus a regimen of amphotericin B followed by fluconazole for candidemia in non-neutropenic patients: A randomized non-inferiority trial. Lancet 2005;366:1435-42.

21. Ministry of Health and Long-Term Care. Ontario Schedule of Benefits for Physicians Services, October 1, 2005. Government of Ontario, Toronto, Ontario.

22. Briggs $\mathrm{AH}$. Handling uncertainty in cost-effectiveness models. Pharmacoeconomics 2000;17:479-500.

23. Bruynesteyn K, Grant V, McKenzie C, et al. A cost-effective analysis of caspofungin vs. liposomal amphotericin B for the treatment of suspected fungal infections in the UK. Eur J Haematol 2007;78:532-9

24. Viscusi WK, Aldy JE. The value of a statistical life: A critical view of market estimates throughout the world. J Risk Uncertain 2003;27:5-76.

25. Bow EJ. Of yeasts and hyphae: A hematologist's approach to antifungal therapy. Hematology Am Soc Hematol Educ Program 2006;361-7.

26. PPS Pharma 2002. PPS Buyers Guide in PPS Pharma 2002. <http://www.ppspharma.com> (Version current at April 29, 2008).

27. Alberta Health and Wellness. Alberta Health and Wellness Drug Benefit List, October 1, 2005. <http://health.gov.ab.ca/ahcip/ ahcip_list.html> (Version current at April 29, 2008).

28. Ministry of Health and Long-Term Care. Drug Benefit Formulary/Comparative Drug Index 2005 (Edition 39). Government of Ontario, Toronto, Ontario. 


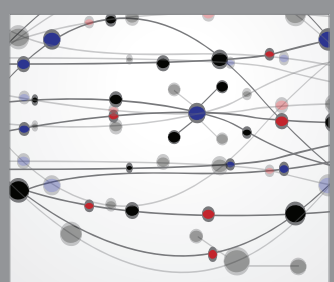

The Scientific World Journal
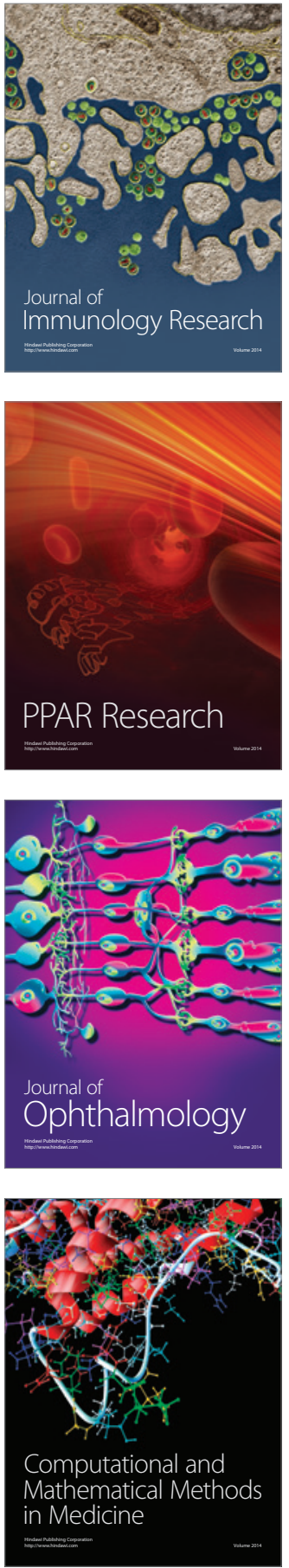

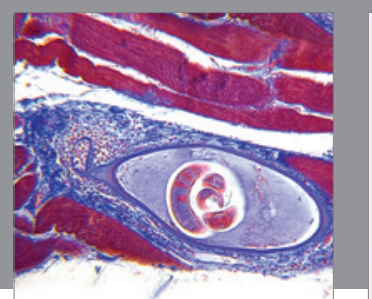

Gastroenterology Research and Practice

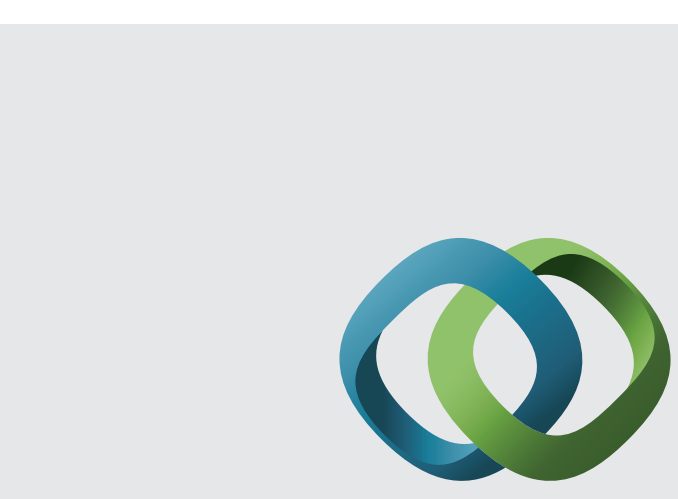

\section{Hindawi}

Submit your manuscripts at

http://www.hindawi.com
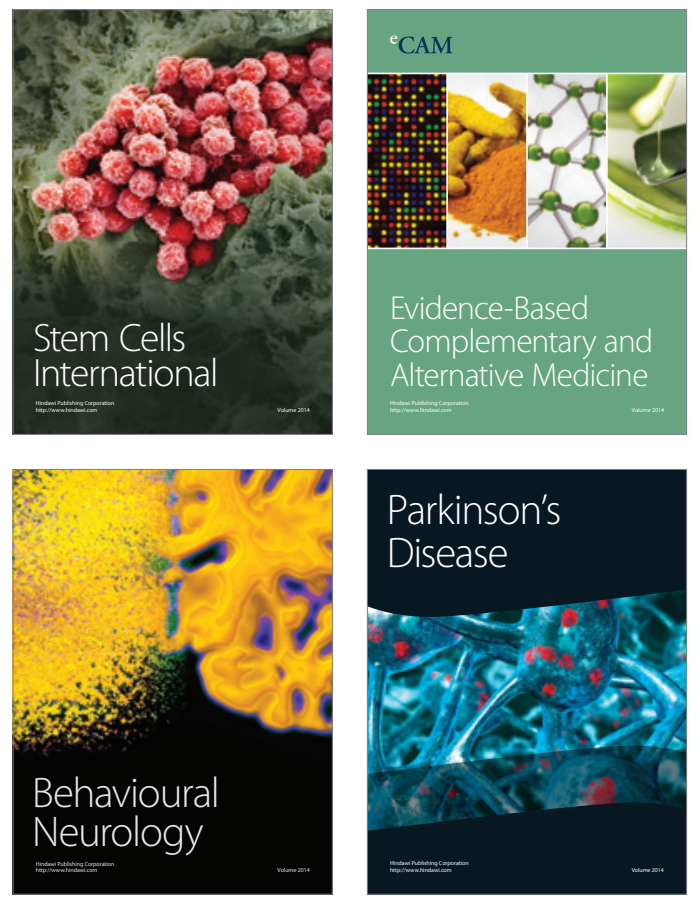
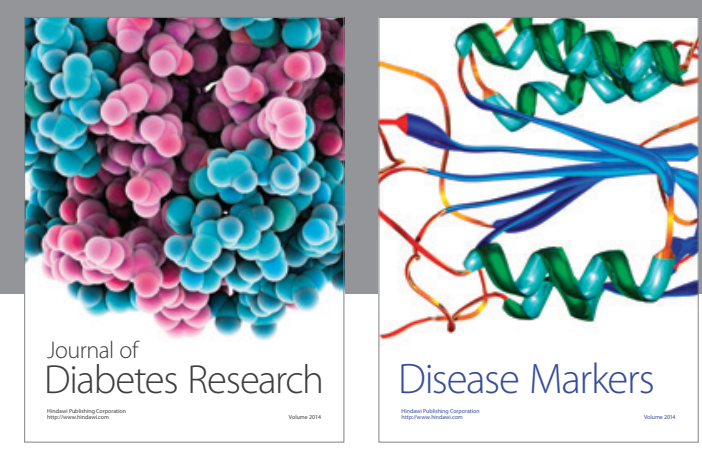

Disease Markers
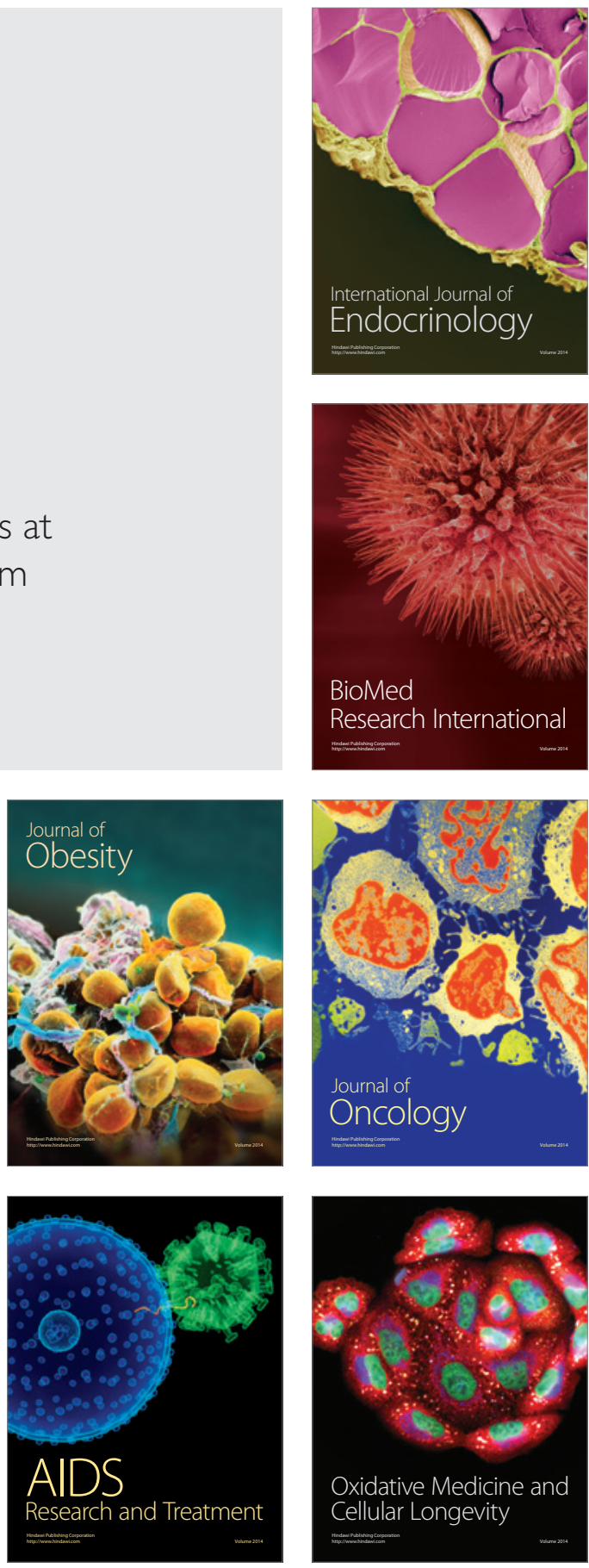